\title{
Effets du climat et des interventions de santé publique sur la pandémie de COVID-19 : une étude de cohorte prospective
}

\author{
Peter Jüni MD, Martina Rothenbühler PhD, Pavlos Bobos MSc, Kevin E. Thorpe MMath, Bruno R. da Costa PhD, \\ David N. Fisman MD, Arthur S. Slutsky MD, Dionne Gesink PhD
}

" Citation : CMAJ 2020 May 25;192:E566-73. doi: 10.1503/cmaj.200920-f; diffusion hâtive le 8 mai 2020

Voir la version anglaise de l'article ici : www.cmaj.ca/lookup/doi/10.1503/cmaj.200920

\section{RÉSUMÉ}

CONTEXTE : On ignore si les variations climatiques saisonnières, la fermeture des établissements scolaires ou d'autres interventions de santé publique entraîneront un ralentissement de la pandémie actuelle de maladie à coronavirus 2019 (COVID-19). Nous avons voulu déterminer si de façon globale la progression de l'épidémie est associée au climat ou aux interventions de santé publique visant à réduire la transmission du coronavirus du syndrome respiratoire aigu sévère 2 (SRAS-CoV-2).

MÉTHODES : Nous avons procédé à une étude de cohorte prospective des 144 régions géopolitiques de la planète (375609 cas) présentant au moins 10 cas de COVID-19, avec transmission locale, en date du 20 mars 2020, à l'exclusion de la Chine, de la Corée du Sud, de l'Iran et de l'Italie. Par analyse de régression à effets aléatoires pondérée, nous avons évalué le lien entre la progression de l'épidémie (exprimée sous forme de rapports de taux d'incidence [RTI] comparant les nombres cumulatifs de cas de COVID-19 du 27 mars 2020 à ceux du 20 mars 2020) avec les facteurs de latitude, température, humidité, fermeture des établissements scolaires, interdiction des grands rassemblements et mesures d'éloignement social qui étaient en place les 7 et 13 mars 2020 (période de 14 jours antérieure à l'évaluation).

RÉSULTATS : Les analyses univariées ont révélé aucuns lien entre la progression de l'épidémie et les facteurs de latitude et de température, mais des liens négatifs faibles avec l'humidité relative (RTI par 10\%, 0,91, intervalle de confiance [IC] de $95 \% 0,85-0,96)$ et l'humidité absolue (RTI par $5 \mathrm{~g} / \mathrm{m}^{3} 0,92$, IC à
$95 \% 0,85-0,99)$. Des liens étroits ont été observés avec l'interdiction des grands rassemblements (RTI 0,65, IC à $95 \%$ 0,53-0,79), la fermeture des établissements scolaires (RTI 0,63, IC à 95\% 0,520,78 ) et les mesures d'éloignement social (RTI 0,62, IC à $95 \% 0,45-0,85$ ). Dans un modèle multivarié, on a noté un lien étroit avec le nombre de mesures déployées par la santé publique ( $p$ pour tendance $=0,001$ ), tandis que le lien avec l'humidité absolue s'atténuait.

INTERPRÉTATION : La progression de l'épidémie de COVID-19 ne s'est pas révélée en lien avec la latitude ni avec la température, mais faiblement en lien avec l'humidité relative ou absolue. À l'inverse, les interventions de santé publique ont été étroitement associées à un ralentissement de la progression de l'épidémie.

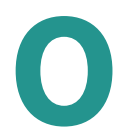

$\mathrm{n}$ connaît bien la saisonnalité de la grippe et sa corrélation avec certains facteurs météorologiques. Les mécanismes avancés pour expliquer le ralentissement des épidémies de grippe durant les mois d'été sous les climats tempérés sont les augmentations de température, d'humidité et d'ensoleillement ${ }^{1}$. Ces 3 facteurs sont liés à la latitude géographique, un élément qu'il est facile de déterminer avec précision. Une autre explication possible du ralentissement des épidémies de grippe durant les mois d'été est la fermeture des établissements scolaires pour les vacances estivales ${ }^{2-4}$.

Dans le but de ralentir la progression de la pandémie actuelle de maladie à coronavirus 2019 (COVID-19), de nombreux pays ont fermé les établissements scolaires ${ }^{5}$ et appliqué d'autres mesures de santé publique, comme l'interdiction des grands rassemblements, l'éloignement social ou la fermeture des commerces non essentiels. Mais on ignore l'effet de ces interventions et des changements météorologiques saisonniers ${ }^{6}$ sur la pandémie. Nous avons procédé à une analyse de la progression actuelle de l'épidémie dans les régions géopolitiques affectées par la COVID19 afin de déterminer si elle est influencée par le climat, la fermeture des établissements scolaires ou les autres interventions de santé publique visant à réduire les contacts dans les populations et ainsi prévenir la transmission du coronavirus du syndrome respiratoire aigu sévère 2 (SRAS-CoV-2), à l'origine de la pandémie ${ }^{7-9}$. 


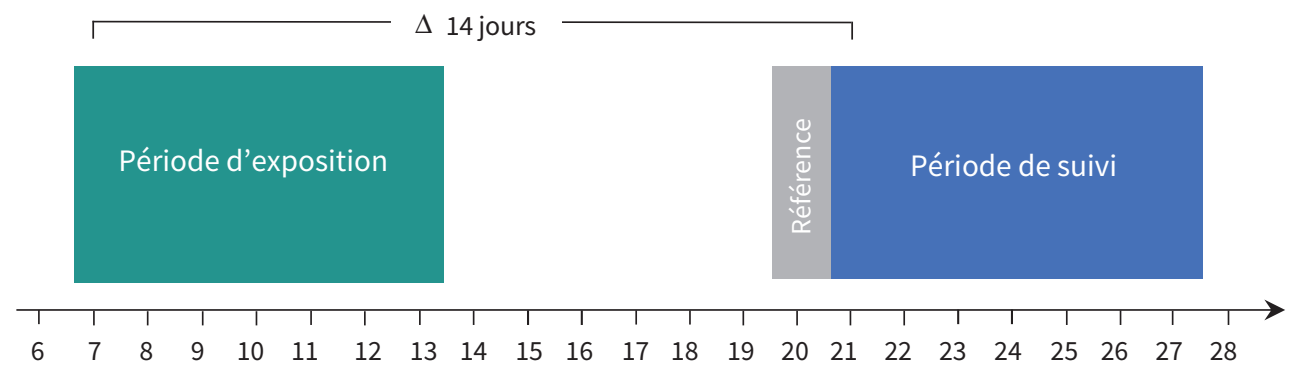

Mars 2020

Figure 1 : Conception de l'étude. $\Delta$ = différence entre le jour 1 de la période d'exposition et le jour 1 de la période de suivi.

\section{Méthodes}

Nous avons conçu une étude de cohorte prospective sur les régions géopolitiques ayant des éclosions documentées de COVID-19 pour déterminer le lien entre la progression de l'épidémie de COVID-19 sur une période de suivi préspécifiée (du 21 au 27 mars 2020) et certains facteurs en place pendant une période antérieure d'exposition couvrant 14 jours (semaines du 7 et du 13 mars 2020). L'écart entre l'exposition et le suivi a été établi à 14 jours, pour faire écho à l'intervalle de temps présumé entre la transmission du SRAS-CoV- $2^{10}$ et le signalement des cas confirmés de COVID-19 (figure 1$)^{11}$. Les analyses ont été effectuées selon un protocole préspécifié. Les résultats d'une analyse préliminaire non publiée, réalisée conformément à la version 1.0 du protocole, sont résumés dans la version $1.2 \mathrm{du}$ protocole (accessible en anglais à l'annexe 1, à l'adresse www.cmaj.ca/ lookup/suppl/doi:10.1503/cmaj.200920/-/DC1). Une explication des changements au protocole est fournie dans la méthodologie supplémentaire, à l'annexe 2, accessible en anglais au www. cmaj.ca/lookup/suppl/doi:10.1503/cmaj.200920/-/DC1.

\section{Admissibilité}

Nous avons inclus toutes les régions géopolitiques (les États de l'Australie et des États-Unis, les provinces et territoires du Canada, les pays et départements ou territoires d'outre-mer pour les autres régions) ayant dénombré au moins 10 cas en date du 20 mars 2020 (valeur de référence) et déclaré une transmission locale avérée selon le Rapport de la situation no 61 de l'Organisation mondiale de la santé (OMS) ${ }^{12}$. La Chine a été exclue, car la progression de son épidémie avait ralenti et l'éclosion semblait contenue. La Corée du Sud, l'Italie et l'Iran ont été exclus, car leurs épidémies étaient clairement établies, la courbe épidémique dans ces territoires étant plus avancée que dans le reste du monde, avec possibilité d'atteinte d'un stade hyperendémique pendant la période de suivi.

\section{Exposition}

La période d'exposition d'une durée préspécifiée allait du 7 au 13 mars 2020 (figure 1). La latitude géographique a été préétablie comme variable d'exposition primaire, tandis que les variables d'exposition secondaires étaient : température moyenne, humidité absolue, fermeture des établissements scolaires, interdiction des grands rassemblements et mesures d'éloignement social. Les données de latitude, de température moyenne, et d'humidité relative moyenne (pour obtenir l'humidité absolue) ont été recueillies pour la capitale de chaque région géopolitique, et les données sur la fermeture des établissements scolaires, l'interdiction des grands rassemblements et les mesures d'éloignement social ont été recueillies à l'échelle des régions géopolitiques. L'humidité absolue représente la teneur absolue en eau en $\mathrm{g} / \mathrm{m}^{3}$, et l'humidité relative représente l'humidité absolue en pourcentage par rapport à l'humidité maximale possible selon la température qu'il fait. Nous avons donné préséance à l'humidité absolue plutôt qu'à l'humidité relative puisqu'elle est en lien plus étroit avec la grippe que l'humidité relative ${ }^{1}$ et a montré moins de variation que l'humidité relative; mais nous avons inclus l'humidité relative comme variable d'exposition post hoc. La température et l'humidité moyennes ont été calculées pour l'entière période d'exposition, générant des moyennes arithmétiques pour toutes les étapes de mesure disponibles (nombre médian 8 par jour, étendue interquartile [EI] de 8 à 45) ${ }^{13}$. Quant à la fermeture des établissements scolaires, l'interdiction des grands rassemblements et l'éloignement social, nous avons déterminé si elles avaient été appliquées à partir d'une date préspécifiée, au milieu d'une semaine de travail de la période d'exposition (le mercredi 11 mars 2020).

\section{Paramètre}

L'analyse des cas confirmés ${ }^{11}$ est compliquée du fait que des modes potentiellement très différents de dépistage et de signalement des individus infectés par le SRAS-CoV-2 ${ }^{14}$ empêchent l'analyse rigoureuse des taux absolus d'événements entre les pays. À l'inverse, une analyse de la progression de l'épidémie ${ }^{15}$ qui peut être exprimée en termes relatifs - un taux d'incidence comparant le nombre cumulatif actuel de cas signalés au nombre cumulatif de cas signalés une semaine plus tôt - risque d'expliquer une certaine variation dans le dépistage et le signalement. Cette approche analyse la courbe de fréquence cumulée 
plutôt que les taux absolus, chaque région géopolitique étant comparée à elle-même (annexe 2, figure S1). La durée préspécifiée de la période de suivi était du 21 au 27 mars 2020 (figure 1). Le paramètre préspécifié était la progression de l'épidémie, définie par un taux d'incidence comparant le nombre cumulatif de cas de COVID-19 confirmés à la fin de la période de suivi le 27 mars 2020, au nombre cumulatif obtenu une semaine auparavant, le 20 mars 2020 (valeur de référence).

\section{Autres covariables}

D’autres covariables étaient préspécifiées : altitude, produit intérieur brut $(\mathrm{PIB})$ per capita, dépenses en santé en pourcentage du PIB, espérance de vie, pourcentage de la population âgée de 65 ans ou plus, indice de vulnérabilité aux maladies infectieuses ${ }^{16}$, densité des populations urbaines, nombre de passagers aériens per capita et distance du plus proche foyer épidémique avéré (ville de Wuhan, Corée du Sud, Iran, Italie). Le tableau S1 de l'annexe 2 explique le choix de ces covariables.

\section{Collecte de données}

Le tableau S1 de l'annexe 2 cite les sources des données. Le 28 mars 2020, nous avons téléchargé les données sur l'éclosion de COVID-19 jusqu'au 27 mars 2020 à partir du tableau de bord interactif en ligne hébergé par le Center for Systems Science and Engineering de l'Université Johns Hopkins, à Baltimore ${ }^{11}$. Le tableau de bord recense quotidiennement le nombre cumulatif de cas à l'échelle des provinces de Chine, à l'échelle des villes ou des comtés de l'Australie, du Canada et des États-Unis; et à l'échelle des pays et des départements ou territoires d'outre-mer ailleurs ${ }^{11}$. Le nombre de cas inscrit au tableau de bord concorde avec les rapports quotidiens compilés par l'OMS ${ }^{11,12}$. Les données rapportées à l'échelle des villes ou des comtés pour l'Australie, le Canada et les États-Unis ont été agrégées par État ou province. Les départements d'outre-mer, comme La Réunion ou les territoires d'outre-mer comme Guam, ont été traités séparément des nations mères aux fins de la présente étude.

La température en degrés Celsius $\left({ }^{\circ} \mathrm{C}\right)$ et l'humidité relative ont été colligées pour la période d'exposition du 7 au 13 mars 2020, à partir d'un site Web météorologique accessible au public ${ }^{13}$, et l'humidité absolue a été calculée à partir de l'humidité relative et de la température à chaque étape de mesure prévue ${ }^{17}$. Les données sur la fermeture des établissements scolaires ont été obtenues de l'Organisation des Nations Unies pour l'éducation, la science et la culture (UNESCO) et de renseignements sur les congés prévus aux calendriers scolaires. Les données sur les congés scolaires, l'interdiction des grands rassemblements et les mesures d'éloignement social ont été obtenues par 1 de 4 investigateurs (P.J., P.B., D.G. et une assistante de recherche) à partir des calendriers scolaires officiels, des directives et communiqués de presse des instances administratives et gouvernementales concernées, ainsi que d'articles de journaux, et vérifiées par au moins un autre investigateur (P.J. ou P.B.). Les données sur l'interdiction des grands rassemblements et les mesures d'éloignement social ont par la suite été vérifiées par rapport aux échéanciers décrits dans l'encyclopédie en ligne Wikipédia ${ }^{19}$. Aucun document n'a été exclu sur la base de la langue. Les membres de l'équipe ont été capables de lire les documents directement dans les langues suivantes: anglais, allemand, tchécoslovaque, danois, néerlandais, français, grec, italien, portugais, slovaque et espagnol. Nous avons utilisé des services de traduction en ligne pour les autres langues. «Éloignement social » désignait toute mesure visant à prévenir les petits rassemblements de 10 personnes ou moins, prenant la forme de recommandations fermes ou d'ordres formels d'éloignement

\begin{tabular}{|c|c|c|}
\hline Variables & $\begin{array}{l}\text { Médiane ou } \\
n\end{array}$ & (EI ou \%) \\
\hline $\mathrm{N}^{\text {bre }}$ de cas & 558 & $221-1419$ \\
\hline $\mathrm{N}^{\text {bre }}$ de cas (par 1000000 d'habitants) & 87.6 & 31.4-193.7 \\
\hline Taux d'incidence & 3.56 & $2.41-4.66$ \\
\hline Latitude (degrés) & 38.4 & $21.8-44.6$ \\
\hline Température $\left({ }^{\circ} \mathrm{C}\right)$ & 12.8 & $7.3-21.2$ \\
\hline Humidité relative (\%) & 69.0 & $60.3-76.6$ \\
\hline Humidité absolue $\left(\mathrm{g} / \mathrm{m}^{3}\right)$ & 7.1 & $5.2-10.8$ \\
\hline Altitude (m) & 82.5 & $16.0-274.0$ \\
\hline Passagers aériens (passagers per capita/an) & 2.3 & $1.0-4.7$ \\
\hline Densité urbaine (1000 habitants $/ \mathrm{km}^{2}$ ) & 3.6 & $1.8-6.2$ \\
\hline Population (1000000 d'habitants) & 7.1 & $3.1-20.6$ \\
\hline $\begin{array}{l}\text { Pourcentage d'habitants âgés de } 65 \text { ans et } \\
\text { plus }\end{array}$ & 14.0 & $8.3-17.2$ \\
\hline Espérance de vie à la naissance, ans & 79 & $76-81$ \\
\hline PIB (1000 \$ US/habitant) & 40.1 & $8.4-56.3$ \\
\hline Dépenses en santé en \% du PIB & 9.2 & $6.3-13.5$ \\
\hline $\begin{array}{l}\text { Indice de vulnérabilité aux maladies } \\
\text { infectieuses }\end{array}$ & 0.87 & $0.64-0.92$ \\
\hline Toute intervention de santé publique & 38 & $26.4 \%$ \\
\hline Interdiction des grands rassemblements & 24 & $16.7 \%$ \\
\hline Éloignement social & 10 & $6.9 \%$ \\
\hline Fermeture des établissements scolaires & 25 & $17.4 \%$ \\
\hline \multicolumn{3}{|l|}{$\mathrm{N}^{\text {bre }}$ d'interventions de santé publique } \\
\hline 0 & 106 & $73.6 \%$ \\
\hline 1 & 24 & $16.7 \%$ \\
\hline 2 or 3 & 14 & $9.7 \%$ \\
\hline \multicolumn{3}{|l|}{ Région du globe } \\
\hline Asie & 30 & $20.8 \%$ \\
\hline Océanie & 6 & $4.2 \%$ \\
\hline Europe & 36 & $25.0 \%$ \\
\hline Afrique & 10 & $6.9 \%$ \\
\hline Amériques & 62 & $43.1 \%$ \\
\hline $\begin{array}{l}\text { Distance du plus proche foyer épidémique } \\
\text { avéré (1000 km) }\end{array}$ & 4.3 & $1.3-8.0$ \\
\hline
\end{tabular}


social, de fermetures des salles à manger des restaurants, des bars et des commerces, à l'exception des épiceries.

Nous avons calculé le nombre de passagers aériens per capita à partir des statistiques publiées à cet égard par les principaux aéroports ${ }^{20-22}$. Les données sur les plus fortes densités urbaines des grandes régions métropolitaines d'une zone géopolitique ont été obtenues de Demographia World Urban Areas $^{23}$, avec des données additionnelles du Bureau du recensement américain ${ }^{24}$. Quant aux données sur les autres covariables, elles proviennent de la Banque mondiale ${ }^{25}$. L'altitude, la température et l'humidité ont été recueillies pour la capitale de chaque région géopolitique, et les autres covariables, à l'échelle des régions géopolitiques. Pour l'Équateur, nous avons recueilli les données émanant de sa capitale économique, Guayaquil ${ }^{25}$. L'indice de vulnérabilité aux maladies infectieuses ${ }^{16}$ n'était disponible qu'à l'échelle des pays; par conséquent, les valeurs propres aux nations mères ont été assignées à leurs États, provinces et départements ou territoires d'outre-mer. Pour rendre l'interprétation de l'indice plus intuitive, nous l'avons inversée de manière à ce que les valeurs plus élevées correspondent à une plus grande vulnérabilité aux maladies infectieuses. Toutes les données ont été complétées au moyen de renseignements accessibles au public - pour les départements ou territoires d'outre-mer, les États ou les provinces des États-Unis, de l'Australie et du Canada - et dans les cas où les données étaient manquantes ou peu plausibles pour les bases de données utilisées au moyen des plus récents renseignements disponibles (annexe 2, tableau S1).

\section{Analyse statistique}

Nous avons utilisé l'analyse de régression à effets aléatoires pondérée ${ }^{26}$ pour déterminer le lien entre les taux d'incidence de COVID-19 à l'échelle logarithmique et les variables d'exposition. Les taux d'incidence ont été calculés sous forme de nombre cumulatif de cas confirmés dans une région géopolitique du début de l'épidémie au 27 mars, divisé par le nombre cumulatif de cas confirmés du début de l'épidémie au 20 mars (annexe 2, figure S1). La période d'observation était identique pour toutes les régions. Étant donné que les populations en question étaient nombreuses, elles pouvaient être considérées comme égales lors des 2 étapes et s'annulaient dans le calcul des taux d'incidence. Un taux d'incidence de 2 indique que le nombre de cas d'une région géopolitique a doublé en l'espace d'une semaine. Étant donné que la période d'exposition du 7 au 13 mars se situait près de l'équinoxe du printemps, aucune transformation n'a été nécessaire pour refléter le lien entre le taux d'incidence de COVID-19 à l'échelle logarithmique avec le carré de la latitude. Les liens ont été exprimés sous forme de rapports des taux d'incidence (RTI) par 400 degrés $^{2}$ d'augmentation de la latitude, $5^{\circ} \mathrm{C}$ d'augmentation de la température, $10 \%$ d'augmentation de l'humidité relative, $5 \mathrm{~g} / \mathrm{m}^{3}$ d'augmentation de l'humidité absolue, et sous forme de RTI comparant les zones géopolitiques ayant ou non appliqué des mesures telles que fermeture des établissements scolaires, interdiction des grands rassemblements ou éloignement social. Les unités d'analyses étaient les régions géopolitiques; les taux d'incidence de COVID-19 à l'échelle logarithmique (variable dépendante) et les variables d'exposition (variables indépendantes) étaient définis à l'échelle des régions géopolitiques. Un RTI inférieur à 1 indique qu'une augmentation d'une variable d'exposition continue ou que la mise en place d'interventions de santé publique sont associées à une baisse de la progression de l'épidémie, et un RTI de 0,60 correspond à une réduction relative de $40 \%$ de la progression de l'épidémie.

Nous avons observé les liens entre la progression de l'épidémie et certaines variables d'exposition au moyen d'analyses univariées et de différents modèles et ensembles d'analyses multivariées pour déterminer la robustesse des liens préspécifiés au protocole (voir annexes 1 et 2). Nous avons ensuite conçu 2 modèles multivariés parcimonieux. Pour le modèle 1 , nous avons d'abord priorisé les covariables sur des bases théoriques, puis nous avons utilisé une analyse typologique non supervisée pour la sélection des variables (annexe 2, tableau $\$ 2$ ) 27 ; pour le modèle 2 , nous avons utilisé une sélection pas à pas à rebours des covariables en fonction de la statistique $R^{2}$ ajustée. Nous avons préspécifié que le modèle 1 allait avoir préséance sur le modèle 2 , puisqu'il ne comportait pas de risque de surapprentissage. L'analyse typologique a dégagé un groupe de 3 interventions de santé publique (annexe 2, figure S2). Nous avons donc dérivé a posteriori un paramètre mixte d'exposition à l'une ou l'autre des 3 interventions. De plus, nous avons effectué des tests préspécifiés pour dégager les tendances en fonction du nombre d'interventions de santé publique déployées ( 0,1 ou 2 ou plus), en supposant que les RTI pour le lien entre progression de l'épidémie et fermeture des établissements scolaires, interdiction des grands rassemblements ou éloignement social iraient dans le même sens et seraient d'une ampleur similaire. Nous avons introduit les principales régions géographiques (Asie, Océanie, Europe, Afrique, Amériques) dans les 2 modèles pour suivre la progression géographique de la pandémie. Les analyses ont été effectuées avec les logiciels Stata Release 14 (StataCorp, College Station, TX) et R (R Foundation for Statistical Computing, Vienne, Autriche).

\section{Approbation éthique}

Cette étude n'a pas nécessité l'approbation d'un comité d'éthique de la recherche, car les données utilisées pour toutes les analyses étaient anonymisées, agrégées et accessibles au public.

\section{Résultats}

Nous avons inclus 144 régions géopolitiques totalisant 375609 cas dans nos analyses (annexe 2, figure S3 et tableau S3). Le nombre médian de cas de COVID-19 par million d'habitants pour les 144 régions géopolitiques était de 87,6 (EI 31,9-193,7); le taux d'incidence médian représentant la progression de l'épidémie était de 3,56 (EI 2,41-4,66, tableau 1). La majeure partie des régions géopolitiques se situaient dans l'hémisphère nord, près du niveau de la mer, sous des climats tempérés. La température médiane était de $12,8^{\circ} \mathrm{C}$ (EI 7,3-21,2), l'humidité relative médiane était de 69,0\% (El 60,3-76,6) et l'humidité absolue médiane était de $7,1 \mathrm{~g} / \mathrm{m}^{3}$ (EI 5,1-10,8). La température et, dans une moindre 
mesure, l'humidité absolue étaient étroitement associées au carré de la latitude; l'humidité relative n'y avait pas été associée (annexe 2, figures S4-S6). Dans 38 régions géopolitiques, au moins une intervention de santé publique avait été appliquée en date du 11 mars 2020, 24 régions ayant déployé une mesure $(16,7 \%)$ et 14 régions en ayant déployé 2 ou $3(9,7 \%)$; les autres n'avaient appliqué aucune intervention de santé publique
(73,6\%). Une corrélation a été établie avec le déploiement d'interventions de santé publique (annexe 2, figure S2). Le pourcentage médian de la population de 65 ans et plus était de 14,0\%; l'espérance de vie médiane à la naissance était de 79 ans; en moyenne, 9,2\% du PIB était consacré à la santé (EI 6,3\%-13,5\%); et la distance médiane du plus proche foyer épidémique avéré était de 4300 kilomètres (El 1300-8000; tableau 1).

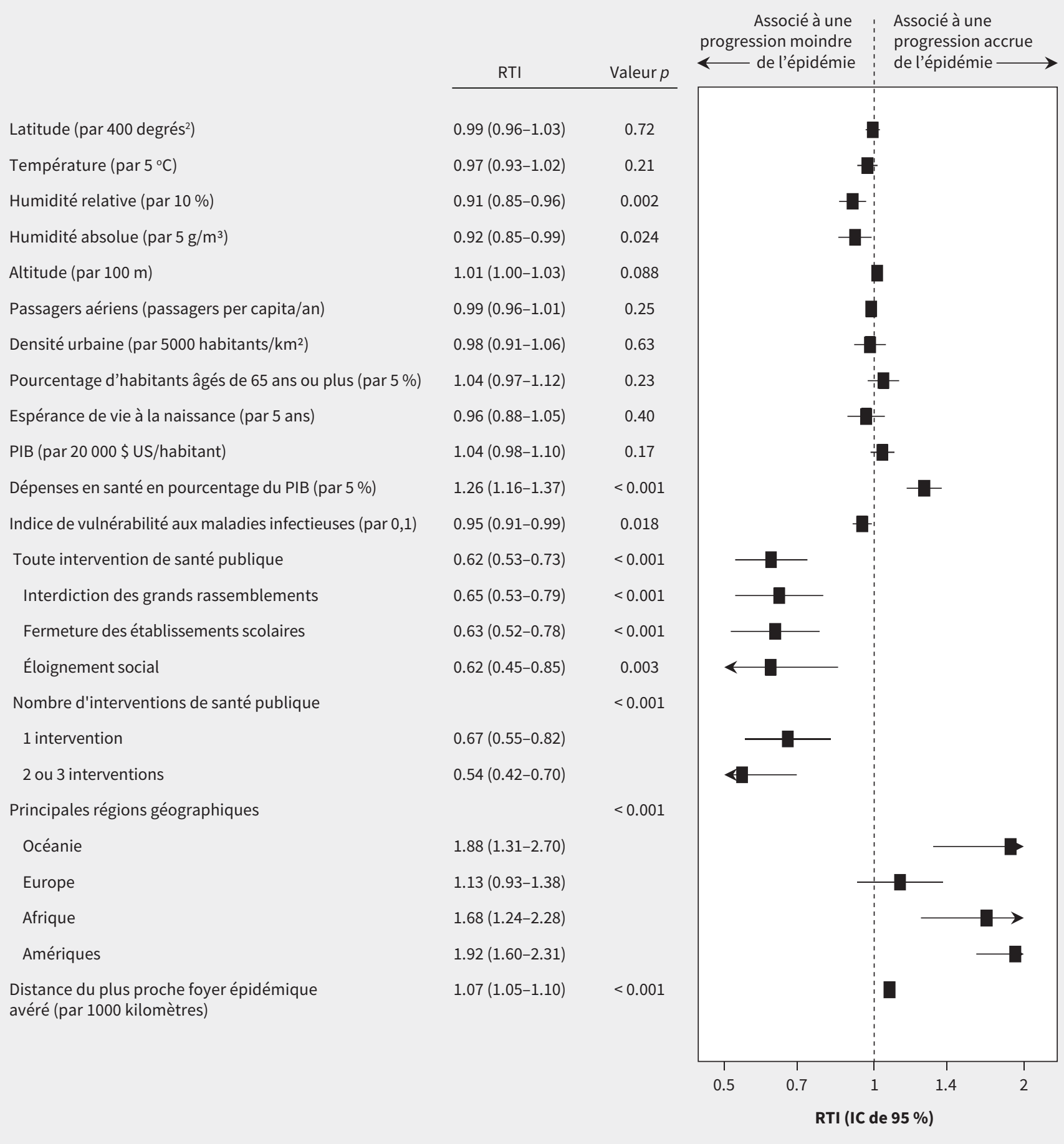

Figure 2 : Résultats des analyses univariées. On y voit les rapports des taux d'incidence (RTI) avec des intervalles de confiance [IC] de $95 \%$ et des valeurs $p$ bilatérales. La valeur $p$ pour le nombre d'interventions de santé publique est une valeur $p$ de tendance. Les catégories de référence sont l'absence d'interventions de santé publique pour le nombre d'interventions de santé publique, et l'Asie pour les principales régions géographiques. Un RTI de 0,62, par exemple, indique une réduction relative de $38 \%$ de la progression de l'épidémie. PIB = produit intérieur brut. 
Dans des analyses univariées (figure 2), on n'a noté aucun lien entre la progression de l'épidémie et la latitude (RTI par 400 degrés ${ }^{2}$ d'augmentation, 0,99 , IC de $95 \%, 0,96-1,03, p=0,72$ ) ou la température moyenne (RTI par $5^{\circ} \mathrm{C}$ d'augmentation 0,97, IC de $95 \%$ 0,93-1,02). À l'inverse, on a noté un lien négatif avec l'humidité relative (RTI par $10 \%$ d'augmentation 0,91 , IC de $95 \%, 0,85-0,96$ ) et l'humidité absolue (RTI par $5 \mathrm{~g} / \mathrm{m}^{3}$ d'augmentation, 0,92, IC de $95 \%, 0,85-0,99)$. À l'annexe 2, les figures S7-S10 montrent au moyen de graphiques à bulles les taux d'incidence de COVID-19 sur une échelle logarithmique en fonction de la latitude, de la température et de l'humidité relative et absolue.

Le paramètre mixte des interventions de santé publique (RTI 0,62, IC de 95\%, 0,53-0,73) et ses différents éléments, soit interdiction des grands rassemblements (RTI de 0,65, IC de 95\%, 0,530,79 ), fermeture des établissements scolaires (RTI de 0,63, IC de $95 \%, 0,52-0,78$ ) et éloignement social (RTI de 0,62, IC de $95 \%$, $0,45-0,85)$ ont tous été en étroite corrélation négative avec la progression de l'épidémie pendant la période de suivi entre le 21 et le 27 mars (annexe 2, figures S11-S13). Le lien négatif était plus prononcé dans les régions géopolitiques appliquant 2 ou 3 interventions de santé publique comparativement aux régions qui n'en appliquaient qu'une seule ( $p$ de tendance $<0,001$; figure 3). La progression de l'épidémie a varié selon les continents, les dépenses en santé, l'indice de vulnérabilité aux maladies infectieuses et la distance du plus proche foyer épidémique avéré.

Dans les analyses multivariées et les analyses limitées préspécifiées, les liens avec la latitude et la température sont demeurés non significatifs (annexe 2, tableaux S4 et S5). Les liens entre progression de l'épidémie et humidité relative et absolue se sont atténués et sont devenus majoritairement non significatifs (annexe 2, tableaux S6 et S7). Les liens négatifs avec les interventions de santé publique sont tous demeurés robustes, à l'exception des mesures d'éloignement social (annexe 2, tableaux S8-S12).

Le modèle multivarié principal (figure 4) a montré un faible lien négatif non significatif entre progression de l'épidémie et humidité absolue (RTI par $5 \mathrm{~g} / \mathrm{m}^{3}, 0,92$, IC de 95\%, 0,84-1,00, $p=$ $0,064)$, mais un lien étroit et constant avec le nombre d'interventions de santé publique déployées ( $p$ de tendance $=$ 0,001). Un modèle multivarié basé sur une sélection pas à pas à rebours (annexe 2, figure S14) a montré un faible lien négatif avec l'humidité absolue (RTI par $5 \mathrm{~g} / \mathrm{m}^{3}$ 0,87, IC de $95 \%, 0,77-$ 0,99 ) et un lien négatif étroit avec le nombre d'interventions de santé publique déployées ( $p$ de tendance $=0,004$ ); il a de plus suggéré un lien négatif avec l'augmentation de l'espérance de vie à la naissance et la variabilité résiduelle par continent. Les analyses post hoc basées sur des paramètres différents pour estimer la progression de l'épidémie ont montré des réductions plus marquées en lien avec les interventions de santé publique (annexe 2, tableaux S13 et S14).

\section{Interprétation}

Dans cette étude de cohorte prospective sur 144 régions géopolitiques regroupant 375609 cas confirmés de COVID-19, la progression de l'épidémie durant la période de suivi du 21 au 27 mars

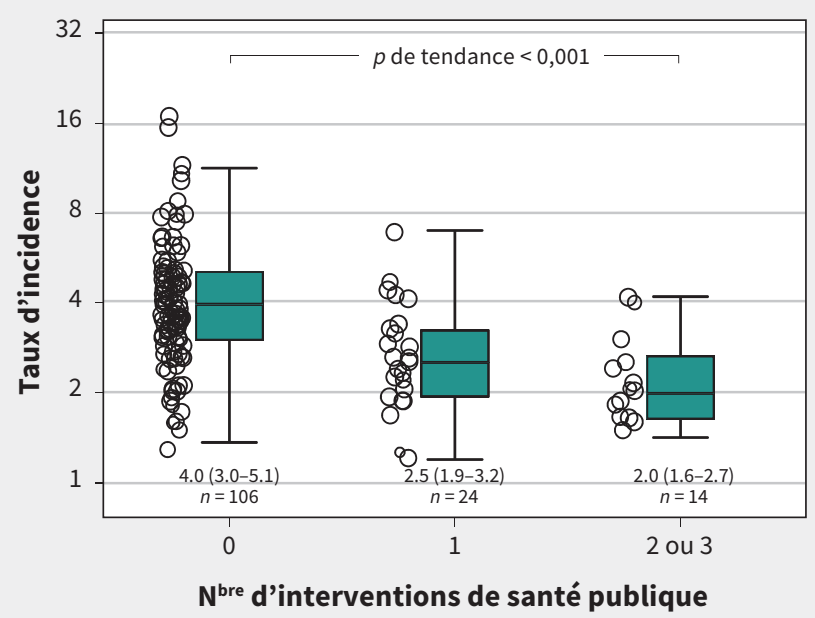

Figure 3 : Progression de l'épidémie par rapport au nombre d'interventions de santé publique ( 0,1 ou 2 ou plus). Chaque bulle représente une région géopolitique, la taille de la bulle étant proportionnelle au poids de la région géopolitique dans un modèle de régression à effets aléatoires pondérée, avec pondération inverse de la variance. Diagramme en boîte à moustaches : la boîte représente l'étendue médiane et interquartile et les moustaches, les valeurs les plus extrêmes de part et d'autre de 1,5 fois l'étendue interquartile au-delà du $25^{\mathrm{e}}$ et du $75^{\mathrm{e}}$ percentile. La valeur $p$ de tendance provient d'une analyse de régression à effets aléatoires pondérée univariée (voir figure 2). Un taux d'incidence de 2 , par exemple, indique que le nombre de cas cumulatifs dans une région géopolitique a doublé en 1 semaine; un taux d'incidence de 3 indique qu'il a triplé.

2020 n'a pas été associée à la latitude géographique, ni à la température qu'il faisait pendant la période d'exposition 14 jours auparavant, où s'est présumément produite la transmission de SRAS-CoV-2. Nous avons observé des liens avec l'humidité relative et absolue, mais ces liens s'amenuisent dans les modèles multivariés. Les liens entre la progression de l'épidémie et les 2 types d'humidité, malgré leur faible corrélation mutuelle ${ }^{28}$, ont suggéré un rôle mineur de l'humidité dans l'épidémiologie de la COVID-19, mais cela demeure hypothétique. Par ailleurs, il est très important que nous ayons observé des liens négatifs étroits avec 3 interventions de santé publique couramment déployées pour contenir la pandémie de COVID-19 : interdiction des grands rassemblements, fermeture des établissements scolaires et éloignement social. Même si nous avons été incapables de quantifier de manière fiable la contribution respective des 3 interventions, nos observations sont pertinentes dans l'immédiat, puisque de nombreux pays envisagent actuellement la levée de certaines de leurs interventions de santé publique.

Nos résultats concordent avec ceux de 3 études chinoises ${ }^{29-31}$ selon lesquelles il n'y avait aucun lien à faire entre la progression de l'épidémie et la température et l'humidité relative ${ }^{29}$, mais de fortes diminutions de la progression de l'épidémie étaient associées aux interventions de santé publique ${ }^{30}$. Dans une récente revue systématique rapide ${ }^{31}$, les données concernant la fermeture des établissements scolaires à l'échelle nationale pour lutter contre la COVID-19 sont très lacunaires et celles qui concernent 
les éclosions de grippe suggèrent que la fermeture des établissements scolaires pourrait avoir des effets relativement mineurs sur le SRAS-CoV-2 en raison de sa grande transmissibilité et de sa faible incidence clinique apparente sur les enfants d'âge scolaire $^{32}$. Selon nos résultats, la fermeture des établissements scolaires aura probablement un effet plus important que ce que suggère cette revue, mais le fait d'avoir amalgamé la fermeture des établissements scolaires avec d'autres interventions de santé publique signifie que nous avons été incapables d'estimer de manière fiable l'effet de cette intervention en particulier sur la pandémie de COVID-19. L'effet de l'interdiction des grands rassemblements, de l'éloignement social et de la fermeture des établissements scolaires sur la transmission virale n'a pas fait l'objet de suffisamment d'études ${ }^{32-34}$. Toutefois, d'après des modèles mathématiques et certaines données d'observation limitées, elles peuvent nuire à la transmission de la maladie. À partir de données mondiales sur la pandémie de COVID-19, notre étude fournit des preuves que ces interventions sont étroitement associées à une réduction de la progression de l'épidémie.

\section{Limites de l'étude}

Notre étude comporte plusieurs limites importantes. Premièrement, en raison de la variabilité considérable des modes de dépistage entre les régions géopolitiques, les taux réels de COVID-
19 n'ont pas pu être estimés de manière fiable. Nous avons toutefois présumé qu'il était possible d'estimer avec un bon degré de fiabilité les taux d'incidence comme mesures de la progression de l'épidémie, étant donné que le mode de dépistage affecterait de la même façon les 2 nombres cumulatifs de cas utilisés pour calculer les taux d'incidence au moment de la période de suivi (1 semaine) préspécifiée. Nous avons été incapables de trouver des données fiables sur le nombre de tests de dépistage du SRASCoV-2 par million d'habitants et sur les différentes stratégies de dépistage, et par conséquent, nous n'avons pas pu vérifier directement cette hypothèse. Les dépenses en santé en pourcentage du PIB et l'indice de vulnérabilité aux maladies infectieuses ${ }^{16}$ pourraient être associés jusqu'à un certain point à la capacité des systèmes de santé d'effectuer un dépistage et servir de marqueurs substituts imparfaits. Les deux ont en effet été associés à la progression de l'épidémie dans l'analyse univariée, mais le modèle multivarié principal n'a pas suggéré de lien avec les dépenses en santé. De plus, les effets aléatoires appliqués au modèle de régression ont implicitement tenu compte de la variabilité résiduelle des caractéristiques des régions géopolitiques qui sont demeurées inexpliquées, y compris la variabilité des stratégies de dépistage. Deuxièmement, nous avons présumé que les stratégies de dépistage du SRAS-CoV-2 ne variaient pas durant la période de suivi. La capacité de dépister était limitée à l'échelle

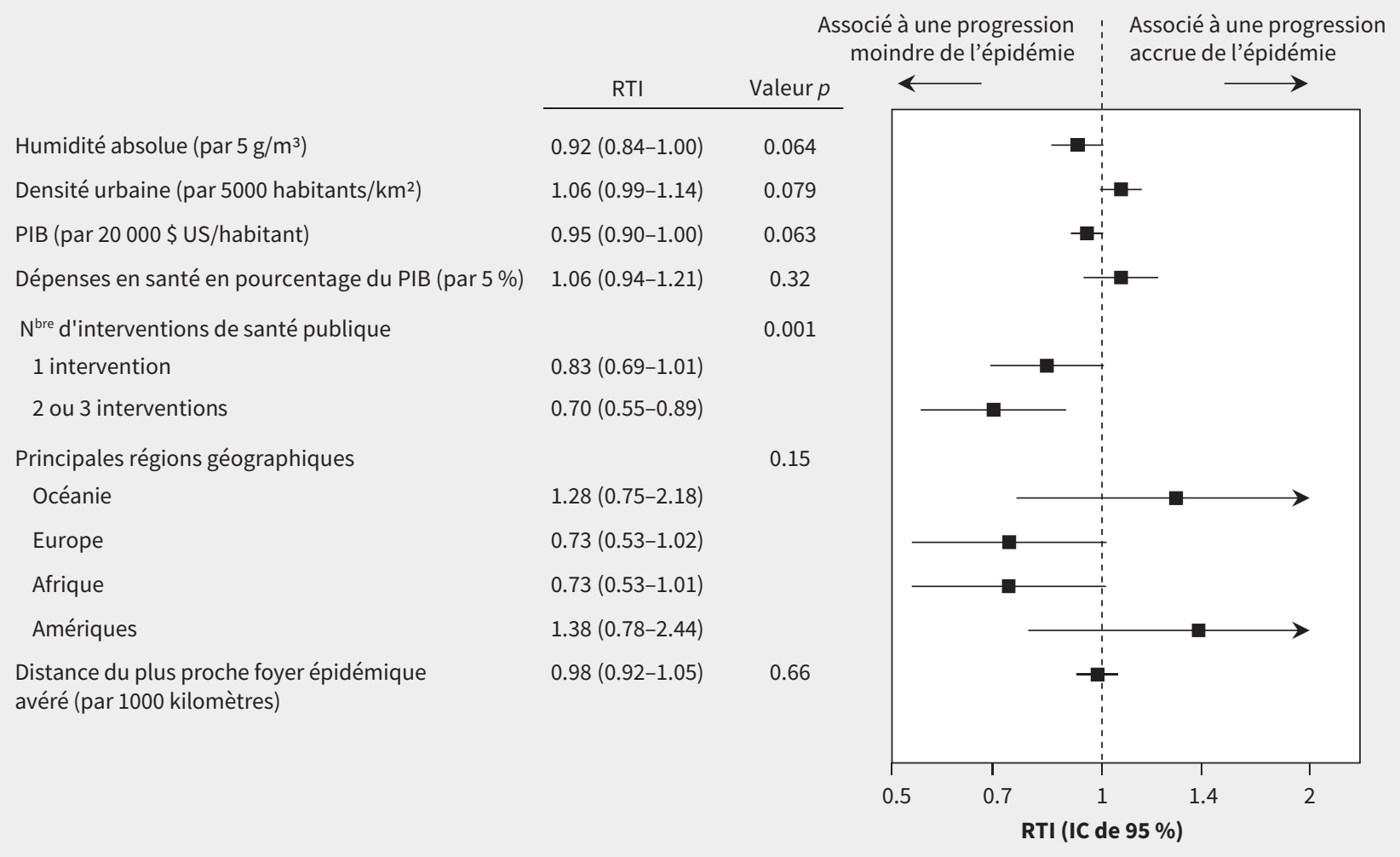

Figure 4 : Résultats du principal modèle multivarié parcimonieux. On y voit les rapports des taux d'incidence (RTI) avec intervalles de confiance [IC] de $95 \%$ et valeurs $p$ bilatérales. Les variables présentées sont celles qui étaient incluses dans le modèle parcimonieux. La valeur $p$ pour le nombre d'interventions de santé publique est une valeur $p$ de tendance. Les catégories de référence sont l'absence d'interventions de santé publique pour le nombre d'interventions de santé publique, et l'Asie pour les principales régions géographiques. Un RTI de 0,70 , par exemple, indique une réduction relative de $30 \%$ de la progression de l'épidémie. PIB = produit intérieur brut. 
mondiale en mars 2020 et risquait peu de changer rapidement durant la période de suivi dans la plupart des régions géopolitiques. En outre, nous croyons que la fenêtre d'une semaine était suffisamment brève pour que les cas confirmés rapportés dans chaque région géopolitique soient susceptibles d'être représentatifs d'un pourcentage constant des cas réels.

Troisièmement, seulement 38 régions géopolitiques avaient appliqué des interventions de santé publique à la date seuil du 11 mars 2020 et ces interventions étaient regroupées. Nous nous sommes donc abstenus d'explorer leur contribution respective et leurs interactions potentielles dans les modèles multivariés et avons simplement conçu une variable mixte binaire, et une variable représentant le nombre d'interventions déployées. Cela signifie que nous avons été incapables d'estimer avec fiabilité la contribution de chacune des 3 interventions de santé publique analysées. Nous avons plutôt tenu compte du lien étroit entre la progression de l'épidémie et le paramètre mixte composé de l'une ou l'autre des interventions de santé publique et de la tendance linéaire du lien avec leur nombre, plus fiable et pertinent que la solidité du lien entre la progression de l'épidémie et les 3 interventions de santé publique prises individuellement. Quatrièmement, on a noté une variabilité des mesures d'éloignement social rapportées par les différentes régions géopolitiques, soit recommandations ou ordres d'éloignement social, fermeture des salles à manger de restaurants et des bars ou fermeture des commerces autres que les épiceries, et le lien moyen qui se dégage ne renseigne pas sur le poids respectif de ces mesures. Cinquièmement, nous avons procédé à l'analyse uniquement lorsque l'interdiction des grands rassemblements a été instaurée, indépendamment de leur taille. Sixièmement, nous avons été incapables de quantifier le degré de conformité de la population à l'égard des mesures d'éloignement social et de l'interdiction des grands rassemblements. À l'inverse, même si on a noté des variations locales quant aux stratégies de fermeture des établissements scolaires, nous considérons que cette mesure est probablement très bien observée.

Septièmement, les données sur la latitude, la température et l'humidité ont été recueillies pour la capitale de chaque région géopolitique, qui n'est peut-être pas entièrement représentative de celles des régions. La granularité limitée des données disponibles peut donc avoir affecté la classification non différentielle de l'exposition, ce qui, en retour, peut avoir biaisé l'estimation des liens vers l'hypothèse nulle (voir annexe 2, tableaux S15-S23 et figure S15 pour plus de détails sur les risques de biais concernant les variables individuelles d'exposition). Le lien entre l'humidité relative et absolue et la progression de l'épidémie a été évoqué, mais inconstant. Même si l'humidité se révèle importante dans l'épidémiologie de la COVID-19 à l'avenir, les effets saisonniers seront probablement atténués par les taux élevés de sensibilité associés aux maladies pandémiques ${ }^{35}$.

\section{Conclusion}

Selon notre analyse globale, la progression de l'épidémie de COVID-19 ne s'est pas révélée en lien avec la latitude géographique ni avec la température durant la période d'exposition. Seules les interventions de santé publique appliquées à l'échelle des régions ont semblé freiner de manière constante la progression de l'épidémie, et le ralentissement a été proportionnel au nombre d'interventions de santé publique déployées simultanément. Prises dans leur ensemble, ces observations suggèrent que les variations climatiques saisonnières ne joueront qu'un rôle minime dans l'épidémiologie de la COVID-19, tandis que les interventions de santé publique (fermeture des établissements scolaires, interdiction des grands rassemblements, éloignement social) pourraient avoir des effets importants. Au moment de décider quand et comment lever les interdictions, l'effet important des interventions de santé publique doit être mesuré avec soin et pris en compte, avec les risques économiques et psychosociaux potentiels.

\section{Références}

1. Tamerius J, Nelson MI, Zhou SZ, et al. Global influenza seasonality: reconciling patterns across temperate and tropical regions. Environ Health Perspect 2011;119:439-45.

2. Cauchemez S, Ferguson NM, Wachtel C, et al. Closure of schools during an influenza pandemic. Lancet Infect Dis 2009;9:473-81.

3. Filleul L, D'Ortenzio E, Kermarec F, et al. Pandemic influenza on Reunion Island and school closure. Lancet Infect Dis 2010;10:294-5.

4. Cauchemez S, Valleron A-J, Boëlle P-Y, et al. Estimating the impact of school closure on influenza transmission from Sentinel data. Nature 2008;452:750-4.

5. Couzin-Frankel J. Does closing schools slow the spread of coronavirus? Past outbreaks provide clues. Science le 10 mars 2020 Accessible ici : www.sciencemag. org/news/2020/03/does-closing-schools-slow-spread-novel-coronavirus (consulté le 7 avril 2020).

6. Cohen J. Why do dozens of diseases wax and wane with the seasons - and will COVID-19? Science le 13 mars 2020 Accessible ici : www.sciencemag.org/news /2020/03/why-do-dozens-diseases-wax-and-wane-seasons-and-will-covid-19 (consulté le 7 avril 2020).

7. Markel H, Lipman HB, Navarro JA, et al. Nonpharmaceutical interventions implemented by US cities during the 1918-1919 influenza pandemic. JAMA 2007;298:644-54.

8. Ferguson N, Laydon D, Nedjati Gilani G, et al. Report 9: Impact of non-pharmaceutical interventions (NPIs) to reduce COVID-19 mortality and healthcare demand. London: Imperial College London; le 16 mars 2020 doi: 10.25561/77482. Accessible ici : http://spiral.imperial.ac.uk/handle/10044/1/77482 (consulté le 6 avril 2020).

9. Bootsma MCJ, Ferguson NM. The effect of public health measures on the 1918 influenza pandemic in U.S. cities. Proc Natl Acad Sci U S A 2007;104:7588-93.

10. Lauer SA, Grantz KH, Bi Q, et al. The incubation period of coronavirus disease 2019 (covid-19) from publicly reported confirmed cases: estimation and application. Ann Intern Med le 10 mars 2020; [Cyberpublication avant impression]. doi: 10.7326/M20-0504.

11. Dong E, Du H, Gardner L. An interactive web-based dashboard to track COVID19 in real time. Lancet Infect Dis 2020;20:533-4.

12. Coronavirus disease 2019 (COVID-19) - situation report 61. Geneva: Organisation Mondiale de la Santé; 2020. Accessible ici : www.who.int/docs/default -source/coronaviruse/situation-reports/20200321-sitrep-61-covid-19.pdf (consulté le 21 mars 2020).

13. Weather for 243 countries of the world. St. Petersburg (Russia): R. Pogodi; 2020. Accessible ici : https://rp5.ru/ (consulté le 14 mars 2020).

14. Zhao S, Musa SS, Lin Q, et al. Estimating the unreported number of novel coronavirus (2019-nCoV) cases in china in the first half of January 2020: a datadriven modelling analysis of the early outbreak. J Clin Med 2020;9. pii: E388. doi: $10.3390 / \mathrm{jcm} 9020388$.

15. Tuite AR, Fisman DN. Reporting, epidemic growth, and reproduction numbers for the 2019 novel Coronavirus (2019-nCoV) Epidemic. Ann Intern Med le 5 février 2020 [Cyberpublication avant impression]. doi: 10.7326/M20-0358. Accessible ici : https:// annals.org/aim/fullarticle/2760912/reporting-epidemic-growth-reproduction -numbers-2019-novel-coronavirus-2019-ncov (consulté le 9 mars 2020).

16. Moore M, Gelfeld B, Okunogbe A, et al. Identifying future disease hot spots: Infectious Disease Vulnerability Index. Santa Monica (CA): Rand Corporation; 2016. Accessible ici : www.rand.org/pubs/research_reports/RR1605.html (consulté le 9 mars 2020).

17. Peci A, Winter A-L, Li Y, et al. Effects of absolute humidity, relative humidity, temperature, and wind speed on influenza activity in Toronto, Ontario, Canada. Appl Environ Microbiol 2019;85.pii: e02426-18. doi: 10.1128/AEM.02426-18. [Sous presse le 15 mars 2019]. 
18. COVID-19 educational disruption and response. Paris (FR): UNESCO; 2020. Accessible ici : https://en.unesco.org/covid19/educationresponse (consulté le 14 mars 2020).

19. Wikipedia. The free encyclopedia. Wikimedia Foundation; 2020. Accessible ici : https://www.wikipedia.org/ (consulté le 12 mars 2020).

20. CAPA - Centre for Aviation. Accessible ici : https://centreforaviation.com/ (consulté le 12 mars 2020).

21. Annual World Airport Traffic Report, 2019. Airports Council International; 2020. Accessible ici : https://store.aci.aero/product/annual-world-airport-traffic -report-2019/ (consulté le 12 mars 2020).

22. Federal Aviation Administration. Accessible ici : www.faa.gov/ (consulté le 12 mars 2020).

23. Demographia World Urban Areas. 15th ed. St. Louis (MO): W. Cox; 2019. Accessible ici : www.demographia.com/db-worldua.pdf (consulté le 14 mars 2020).

24. United States Census Bureau. Accessible ici : www.census.gov/ (consulté le 12 mars 2020).

25. World development indicators. Washington (D.C.): The World Bank Group; 2020. Accessible ici : https://data.worldbank.org/ (consulté le 9 mars 2020).

26. Harbord RM, Higgins JPT. Meta-regression in Stata. Stata J 2008;8:493-519.

27. Harrell FE, Lee KL, Califf RM, et al. Regression modelling strategies for improved prognostic prediction. Stat Med 1984;3:143-52.

28. Nguyen JL, Schwartz J, Dockery DW. The relationship between indoor and outdoor temperature, apparent temperature, relative humidity, and absolute humidity. Indoor Air 2014;24:103-12.
29. Yao Y, Pan J, Liu Z, et al. No Association of COVID-19 transmission with temperature or UV radiation in Chinese cities. Eur Respir J le 8 avril 2020 [Cyberpublication avant impression]. pii: 2000517. doi: 10.1183/13993003.00517-2020.

30. Pan A, Liu L, Wang C, et al. Association of public health interventions with the epidemiology of the COVID-19 outbreak in Wuhan, China. JAMA le 10 avril 2020 [Cyberpublication avant impression]. doi: 10.1001/jama.2020.6130. Accessible ici : https://jamanetwork.com/journals/jama/fullarticle/2764658 (consulté le 13 avril 2020).

31. Leung K, Wu JT, Liu D, et al. First-wave COVID-19 transmissibility and severity in China outside Hubei after control measures, and second-wave scenario planning: a modelling impact assessment. Lancet 2020;395:1382-93.

32. Viner RM, Russell SJ, Croker $\mathrm{H}$, et al. School closure and management practices during coronavirus outbreaks including COVID-19: a rapid systematic review. Lancet Child Adolesc Health 2020;4:397-404.

33. Park M, Cook AR, Lim JT, et al. A systematic review of COVID-19 epidemiology based on current evidence. J Clin Med 2020;9:pii:E967.

34. Nunan D, Brassey J. What is the evidence for mass gatherings during global pandemics? A rapid summary of best-available evidence. Oxford (UK): The Centre for Evidence-Based Medicine: Oxford COVID-19 Evidence Service. Accessible ici : www.cebm.net/wp-content/uploads/2020/03/Mass -gatherings-and-sporting-events-during-a-pandemic_PDF-template-4.pdf (consulté le $1^{\text {er }}$ mai 2020).

35. Simonsen L, Chowell G, Andreasen V, et al. A review of the 1918 herald pan demic wave: importance for contemporary pandemic response strategies. Ann Epidemiol 2018;28:281-8.

\section{Intérêts concurrents : Aucun déclaré.}

Cet article a été révisé par des pairs.

Affiliations : Centre de recherche en santé appliquée (Jüni, Rothenbühler, Bobos, Thorpe, da Costa, Slutsky) Institut du savoir Li Ka Shing, Hôpital St. Michael; Département de médecine et Institut des politiques, de la gestion et de l'évaluation de la santé (Jüni), Université de Toronto, Toronto, Ont.; Ava AG (Rothenbühler), Zürich, Suisse; Département des sciences de la santé et de la réadaptation (Bobos), Université Western, London, Ont.; École Dalla Lana de santé publique (Thorpe, Fisman, Gesink), Université de Toronto, Toronto, Ont.; Institut des soins de santé primaires (da Costa), Université de Berne, Suisse; Division interdépartmentale de médecine de soins intensifs (Slutsky), Université de Toronto, Toronto, Ont.

Collaborateurs : Peter Jüni a conçu et élaboré l'étude, recueilli, analysé et interprété les données, rédigé la première ébauche de l'article et contribué à toutes les révisions. Martina Rothenbühler et Bruno da Costa ont analysé et interprété les données et contribué à toutes les révisions. Pavlos Bobos a contribué à la conception de l'étude, recueilli et interprété les données et participé à toutes les révisions. Kevin Thorpe a analysé et interprété les données et contribué à toutes les révisions. David Fisman et Arthur Slutsky ont contribué à la conception de l'étude, à l'interprétation des données et à toutes les révisions. Dionne Gesink a contribué à la conception de l'étude, recueilli et interprété les données, rédigé la première ébauche de l'article et participé à toutes les révisions. Tous les auteurs ont donné leur approbation finale pour la version soumise pour publication et assument l'entière responsabilité de tous les aspects du travail.

Financement : Peter Jüni est détenteur d'une Chaire de recherche du Canada de niveau 1 en épidémiologie clinique des maladies chroniques. Pavlos Bobos est bénéficiaire de bourses doctorales FrederickBanting et Charles-Best des Instituts de recherche en santé du Canada. Arthur S. Slutsky a reçu les subventions nos FDN143285 et 137772 des Instituts de recherche en santé du Canada. Cette recherche a été complétée en partie grâce à un financement du Programme des chaires de recherche du Canada et des Instituts de recherche en santé du Canada.

Partage des données : Les auteurs accueillent favorablement les propositions d'utilisation conjointe des données de l'étude. Les propositions d'utilisation conjointe des données de l'étude devraient être envoyées à l'auteur de correspondance (peter.juni@utoronto.ca). Les données seront accessibles avec le soutien des investigateurs moyennant la signature d'une entente pour l'accès aux données, après approbation de la proposition par les investigateurs de l'étude.

Remerciement : Les auteurs remercient Maggie Law pour son aide à la collecte des données concernant les interventions de santé publique.

Accepté : le 5 mai 2020

Correspondance : Peter Jüni, peter.juni@utoronto.ca 